\title{
A Probabilistic Approach for InSAR Time-Series Postprocessing
}

\author{
Ling Chang and Ramon F. Hanssen, Senior Member, IEEE
}

\begin{abstract}
Monitoring the kinematic behavior of enormous amounts of points and objects anywhere on Earth is now feasible on a weekly basis using radar interferometry from Earth-orbiting satellites. An increasing number of satellite missions are capable of delivering data that can be used to monitor geophysical processes, mining and construction activities, public infrastructure, or even individual buildings. The parameters estimated from these data are used to better understand various natural hazards, improve public safety, or enhance asset management activities. Yet, the mathematical estimation of kinematic parameters from interferometric data is an ill-posed problem as there is no unique solution, and small changes in the data may lead to significantly different parameter estimates. This problem results in multiple possible outcomes given the same data, hampering public acceptance, particularly in critical conditions. Here, we propose a method to address this problem in a probabilistic way, which is based on multiple hypotheses testing. We demonstrate that it is possible to systematically evaluate competing kinematic models in order to find an optimal model and to assign likelihoods to the results. Using the B-method of testing, a numerically efficient implementation is achieved, which is able to evaluate hundreds of competing models per point. Our approach will not solve the nonuniqueness problem of interferometric synthetic aperture radar (InSAR), but it will allow users to critically evaluate (conflicting) results, avoid overinterpretation, and thereby consolidate InSAR as a geodetic technique.
\end{abstract}

Index Terms-B-method of testing, deformation modeling, multiple hypotheses testing (MHT).

\section{INTRODUCTION}

$\mathbf{I}$ NTERFEROMETRIC synthetic aperture radar (InSAR) time-series techniques, such as persistent and distributed scatterer (PS/DS) time series [1]-[8], in all their variations, need to disentangle two highly correlated parameter groups as follows: the quality of the time series (coherence) [9], [10] and the parameters of interest, i.e., topographic, kinematic, atmospheric, and phase ambiguity parameters [11], [12]. Focusing on the kinematic parameters, which are often referred to as the deformation, this problem is ill posed and unsolvable since the variability in the time series of every single point (i.e., a PS or equivalent as a group of spatially homogeneous DSs) is equally likely attributed to noise such as thermal noise and

Manuscript received February 5, 2015; revised May 6, 2015; accepted July $19,2015$.

The authors are with the Department of Geoscience and Remote Sensing, Delft University of Technology, $2628 \mathrm{CN}$ Delft, The Netherlands (e-mail: L.Chang@ tudelft.nl).

Color versions of one or more of the figures in this paper are available online at http://ieeexplore.ieee.org.

Digital Object Identifier 10.1109/TGRS.2015.2459037 decorrelation [3], [13], [14], as well as to the actual motion, unless additional constraints can be added.

Quality estimates are required to select which of the billions of reflections in SAR images can be used to estimate the parameters of interest. In particular, the presence of unknown integer phase ambiguities makes this problem cumbersome. Since quality estimates and parameter estimates are not independent, all methods use heuristic solutions for this problem [15], [16].

Practically, InSAR methods use harsh assumptions, i.e., the expected behavior of the signal either in time (e.g., a certain "smoothness" of the kinematic signal) [4], [10], [12] or in space (the smoothness between nearby points) [6], [17]. This is mostly parameterized using the temporal or spatial estimators of coherence. Although there are several classes of problems where some of these assumptions seem to be fair, as the many successful InSAR applications in literature show [18]-[20], in its origin, the dependence on such assumptions makes the techniques extremely vulnerable. This has been the outcome of several studies [21], [22], where different expert groups processed the same data, leading to different results. More specifically, even a single expert can use different processing tools and obtain significant differences in the results using the same input data [23]. Nowadays, this is one of the main bottlenecks for the operational acceptance of InSAR time series by end users who are not radar experts. The other bottleneck in the operational use of InSAR time series is the inability to analyze extremely large data sets showing the kinematic behavior of points. Visualizations in terms of "velocity maps" (essentially an aggregate variable based on the time series) are poor as they do not come close to convey the rich information content in the data.

To address these problems, we start with postulating some guiding principles. First, there is no single assumption on signal behavior that satisfies all InSAR measurement points. Consequently, the processed results of millions of locations will have inhomogeneous qualities. Second, InSAR data are obtained opportunistically, at times dictated by satellite orbits and at positions provided by mere chance based on the size, shape, and orientation of targets. Third, due to the phase ambiguity problem, the estimated parameters are extremely correlated, and there are more solutions given an observed phase time history, with different degrees of likelihood.

Given these postulates, we propose a new probabilistic approach to tackle this problem. The approach is aimed at the postprocessing of estimated InSAR time-series results, but it could be applied at different stages in the processing chain. It consists of two parts. In the first part, the estimated kinematic 
time series are scrutinized as a whole. In this stage, we estimate the noise of the reference point using the per-epoch correlation between all arcs relative to the reference point. In the second part, we apply multiple hypotheses testing (MHT) [24], [25] to find an optimal kinematic model describing the observations. We first establish a library of canonical functions that may be used or combined to build temporal models applicable to the time series of a specific point. We then start by defining a null hypothesis, which is based on the steady-state behavior, and we compute the proper test statistic to decide whether the null hypothesis needs to be rejected. If this is the case, we test the entire library of potential alternative models with different physically realistic parameters against the null hypothesis. We account for the different degrees of freedom (or dimensions) of the various tests, depending on the amount of parameters that need to be estimated. This is based on Baarda's B-method of testing [26]. This method stems from geodetic literature and precedes model order selection methods such as the Akaike information criterion [27], [28] or the Bayesian information criterion (cf., [29] for a review of information criterion rules). Similar to those methods, the B-method of testing penalizes the use of models with an increased number of parameters (to avoid overfitting) while rewarding the goodness of fit of the model, but it achieves this by changing the critical value, above which a hypothesis will be rejected, via its relationship with the likelihood of a type-I error, a noncentrality parameter, and the power of the test (see Section II-B). This ensures that alternative models are selected with the same probability of correct detection, whereas the actual parameters do not need to be estimated explicitly. Then, for computing the test statistics for more than 100 alternative hypotheses, we present a computationally efficient method only using the residuals under the null hypothesis and an a priori defined matrix per alternative hypothesis. This way, it is not necessary to explicitly compute parameter results for each individual alternative hypothesis. Finally, using test ratios, it is possible to select the most likely model for each point and to update the quality description. We can evaluate hundreds of thousands of points within a few minutes on a standard computer.

This paper is organized as follows. In Section II, we introduce the concepts, the methodology, and the applied procedure, followed by the numerical implementation in Section III, the results on simulated and real data in Section IV, and the conclusions in Section V.

\section{Methodology}

We first introduce the concepts of MHT, the B-method of testing, and the detection-identification-adaptation (DIA) procedure [30]. Then, we build a library of canonical functions to model the kinematic behavior of points in SAR data, and we relate this to physically realistic parameters.

\section{A. $M H T$}

Multiple Hypotheses: The mathematical expressions of null hypothesis $H_{0}$ and all possible alternative hypotheses $H_{j}, \forall j$ are given by

$$
\begin{aligned}
& H_{0}: \quad E \underset{m \times 1}{\{\underline{y}\}}=\underset{m \times n}{A} \underset{n \times 1}{x} \\
& D\{\underline{y}\}=\underset{m \times m}{Q_{y y}}=\sigma^{2} R_{y y} \\
& H_{j}: \quad E \underset{m \times 1}{\{\underline{y}\}}=\underset{m \times n}{A} \underset{n \times 1}{x}+\underset{m \times q}{C_{j}} \underset{q \times 1}{\nabla_{j}}, \underset{q \times 1}{\nabla_{j}} \neq 0 \\
& D\{\underline{y}\}=\underset{\substack{Q_{y y} \\
\text { y }}}{Q^{2}}=\sigma^{2} R_{y y}
\end{aligned}
$$

where $A$ is the design matrix, $E\{\cdot\}$ is the expectation operator, $m$ is the number of stochastic observations $\underline{y}$, and $n$ is the number of unknowns $x$. Any alternative hypothesis is defined by a specification matrix $C_{j}$ and an additional new vector of unknown parameters $\nabla_{j}$ of the dimension (or degree of freedom) $q$, with $q \in[1, m-n]$. The dispersion $D$ of the observations is given using the variance of unit weight $\sigma^{2}$ and the cofactor matrix $R_{y y}$ of covariance matrix $Q_{y y}$.

Test Statistic: Since we want to evaluate multiple alternative hypotheses against $H_{0}$, the rejection of $H_{0}$ will not directly lead to the identification of the optimal $H_{j}$ at a requested level of significance $\alpha$. For all $H_{j}$, we need to compare the test statistics to find the optimal statistic, which needs to be determined using a uniform test criterion. Every test follows a test statistic $\underline{T}_{q}$ with a chi-squared distribution $\chi^{2}(q, \lambda)$, where $\lambda$ indicates the level of noncentrality. For every testing operation between the null hypothesis and an alternative hypothesis, the distributions are [24], [25]

$$
H_{0}: \quad \underline{T}_{0} \sim \chi^{2}(q, 0) \quad H_{j}: \quad \underline{T}_{q}^{j} \sim \chi^{2}\left(q, \lambda_{j}\right) \quad \forall j
$$

where

$$
\begin{aligned}
\lambda_{j} & =\nabla_{j}^{T} C_{j}^{T} Q_{y y}^{-1} Q_{\hat{e}_{0} \hat{e}_{0}} Q_{y}^{-1} C_{j} \nabla_{j} \\
Q_{\hat{e}_{0} \hat{e}_{0}} & =Q_{y y}-A\left(A^{T} Q_{y y}^{-1} A\right)^{-1} A^{T}
\end{aligned}
$$

is the covariance matrix of residual $\underline{\hat{e}}_{0}=y-\underline{y}_{0}$ between the observations and estimates $\underline{\hat{y}}_{0}=A\left(A^{T} Q_{y y}^{-1} A\right)^{-1} A^{T} Q_{y y}^{-1} \underline{y}$ under the null hypothesis. Then, $\underline{T}_{q}^{j}$ can be generally expressed as [16]

$$
\begin{aligned}
\underline{T}_{q}^{j} & =\underline{\hat{e}}_{0}^{T} \underbrace{Q_{y y}^{-1} C_{j}\left(C_{j}^{T} Q_{y y}^{-1} Q_{\hat{e}_{0} \hat{e}_{0}} Q_{y y}^{-1} C_{j}\right)^{-1} C_{j}^{T} Q_{y y}^{-1}}_{L^{j}} \underline{\hat{e}}_{0} \\
& =\underline{\hat{e}}_{0}^{T} L^{j} \underline{\hat{e}}_{0} .
\end{aligned}
$$

We introduce the $m \times m$ symmetric matrix $L^{j}$, which is equal for each point to be evaluated, and it only varies per applied model under the alternative hypothesis. Therefore, the set of $L$ matrices needs to be only computed once in the initialization stage, and it can be then efficiently used while evaluating millions of InSAR points.

Test Ratios: When alternative hypotheses have different dimensions, i.e., $q_{i} \neq q_{j}$ and $q_{i}, q_{j} \in[1, m-n]$, their test statistics have different $\chi^{2}$. Consequently, the best model cannot be determined by (5). This problem is solved by dividing the test statistic by its critical value $\chi_{\alpha_{j}}^{2}\left(q_{j}\right)$, with a predefined level of 
significance $\alpha_{j}$, expressed as a test ratio as follows [31]:

$$
\underline{\mathbf{T}}_{q_{j}}^{j}=\underline{T}_{q_{j}}^{j} / \chi_{\alpha_{j}}^{2}\left(q_{j}\right) .
$$

Note that the $\chi^{2}$ distribution is a special case of the Gamma distribution ( $\Gamma$ distribution), i.e., distribution $\underline{T}_{q} \sim \chi^{2}(q, 0)$ is equal to distribution $\underline{T}_{q} \sim \Gamma(q / 2,1 / 2)$. This implies that the multiplication of $\underline{T}_{q} \sim \chi^{2}(q, 0)$ with a factor $C=1 / \chi_{\alpha}^{2}(q)>$ 0 yields Gamma function $\underline{\mathbf{T}}_{q}=C \underline{T}_{q} \sim \Gamma(q / 2,2 C)$ [32], [33].

Whenever ratio $\underline{\mathbf{T}}_{q_{j}}^{j}>1, H_{j}$ is favored over $H_{0}$. When ratio $\underline{\mathbf{T}}_{q_{j}}^{j} \leq 1, H_{j}$ is not more probable than $H_{0}$ and is therefore discarded. The optimal alternative hypothesis, which is denoted as $H_{B}$, is expected to reject $H_{0}$ most significantly; therefore, it must have the largest test ratio. In other words, $H_{B}$ is the most probable alternative hypothesis if and only if we have [31]

$$
\underline{\mathbf{T}}_{q_{B}}^{B}=\max _{j}\left\{\underline{\mathbf{T}}_{q_{j}}^{j}\right\}, \quad \forall j .
$$

The proper setting of the test parameters, such as $\alpha_{j}$, is required in this comparison. We use the B-method of testing as the selection method for the test parameters.

\section{B. B-Method of Testing}

The critical value $\chi_{\alpha_{j}}^{2}\left(q_{j}\right)$ in (6) depends on the choice of the level of significance $\alpha_{j}$ and dimension $q_{j}$. The level of significance refers to the type-I error, i.e., the rejection of $H_{0}$ when in fact $H_{0}$ is true [34]. However, when the aim is to compare different alternative hypotheses, we want to make sure that the probability of accepting a particular alternative hypothesis, in the case where it is true, is identical for any alternative hypothesis. This probability is commonly referred to as the power, i.e., $\gamma$, of the test. The essence of the B-method of testing [26] is to fix the reference power of the test $\gamma_{0}$ and to calculate reference noncentrality parameter $\lambda_{0}$ given the level of significance $\alpha_{j}$ and dimension $q_{j}$, as in

$$
\lambda_{0}=\lambda\left(\gamma_{0}, \alpha_{j}, q_{j}\right), \quad \forall j .
$$

Thus, the constant values for noncentrality parameter $\lambda_{0}$ and power $\gamma_{0}$ for all tests assure that any particular alternative hypothesis $H_{j}$ can be selected with the same probability irrespective of its dimension $q_{j}$.

Furthermore, we can determine the minimal absolute value $\left|\nabla_{j}\right|$ to make the additional parameter vector $\nabla_{j}$ detectable with discriminatory power $\gamma_{0}$. This value is found via the concept of internal reliability for a given level of significance $\alpha_{0}$, power $\gamma_{0}$, and dimension $q$ by [16], [26]

$$
\nabla^{T} C^{T} Q_{y y}^{-1} Q_{\hat{e}_{0} \hat{e}_{0}} Q_{y y}^{-1} C \nabla=\underset{q \times q}{\lambda}(\gamma, \alpha, q) .
$$

If the new parameter vector $\nabla$ is known, discriminatory power (or the power of test) $\gamma$ can be straightforwardly computed via noncentrality parameter $\lambda$. Since power $\gamma$ is the likelihood of correctly rejecting $H_{0}$ in favor of $H_{j}$, a large value for $\nabla$ yields large power, making it easy to detect, whereas a small value for $\nabla$ yields low discriminatory power.
For InSAR kinematic time-series modeling, the objective is to compare various alternative hypotheses $H_{j}$ with the same discriminatory power $\gamma$. Once $\gamma=\gamma_{0}$ and $\alpha_{0}$ are fixed, the corresponding noncentrality parameter $\lambda_{0}=\lambda\left(\gamma_{0}, \alpha_{0}, q\right)$ can be obtained for a certain dimension $q$. Subsequently, for a different alternative hypothesis, i.e., $H_{i}$, with $q_{i}$ degrees of freedom, we now need to adjust $\alpha$ in order to keep the power of the test $\gamma_{0}$ the same.

\section{DIA Procedure}

The proposed testing procedure follows the DIA procedure [30].

1) Detection: In the first part of the DIA procedure, an overall model test (OMT) [25] is applied on the null hypothesis $H_{0}$ of the steady-state (constant velocity) motion. The test statistic for the OMT is

$$
\underline{T}_{0}=\underline{\hat{e}}_{0}^{T} Q_{y y}^{-1} \underline{\hat{e}}_{0} .
$$

The purpose of the OMT is to detect deviations from $H_{0}$ and to consequently decide to either sustain or reject the null hypothesis. However, in the case of rejection, the OMT cannot determine its cause.

2) Identification of Optimal Model: Rejection can result from an inappropriate functional model or the poor specification of the noise signature of the observations in stochastic model $Q_{y y}$. A physically realistic alternative parameterization of the kinematic behavior can be designed. A priori information on the problem at hand or a flexible and expandable library of model functions can be used to build many alternative functional models to later decide on the most probable alternative model by searching the maximum test ratio, as in (6). This will be elaborated in Section II-D.

3) Adaptation: Estimation and Evaluation: Using the identified optimal model, we estimate the augmented vector of unknowns $\hat{x}^{(1)}=[\hat{x}, \hat{\nabla}]^{T}$. We can evaluate its quality in terms of its precision and reliability. The precision of the estimated parameters for every model under one hypothesis is given by the inverse of normal matrix $N$ as follows:

$$
Q_{\hat{x} \hat{x}}=N^{-1}=\left(\left[A \vdots C_{j}\right]^{T} Q_{y y}^{-1}\left[A \vdots C_{j}\right]\right)^{-1} .
$$

Note that we use $\hat{x}$ to replace $\hat{x}^{(1)}$ in (11) for the sake of brevity. This precision indicator $Q_{\hat{x} \hat{x}}$ can be compared with a predefined criterion matrix $\mathbb{H}_{x}$ that is a symmetric and positivedefinite matrix, which is filled with the values derived from a suitable (co)variance function [35]. The estimated precision of the parameters $Q_{\hat{x} \hat{x}}$ satisfies the criterion precision if and only if we have

$$
\underset{n \times n}{Q_{\hat{x} \hat{x}}} \leq \underset{n \times n}{\mathbb{H}_{x}} .
$$

This inequality can be cast in a generalized eigenvalue problem as follows:

$$
\left|Q_{\hat{x} \hat{x}}-\zeta \mathbb{H}_{x}\right|=0
$$


where $|\cdot|$ denotes the determinant, and eigenvalue $\zeta$ scales $\mathbb{H}_{x}$. When the two matrices, i.e., $Q_{\hat{x} \hat{x}}$ and $\mathbb{H}_{x}$, are identical, all eigenvalues are 1 . If the eigenvalues are smaller than 1 , the precision is better than required. If the eigenvalues are larger than 1, the precision is worse than required. Maximum eigenvalue $\zeta_{\max }$ corresponds to the estimated parameters with the worst precision, and it is considered a metric for the global precision.

\section{Library of Canonical Kinematic Models}

In InSAR data processing, the kinematic model for every point at position $\mathbf{x}=\mathbf{x}(x, y)$ can be regarded as unique and uncorrelated to other points. We consider a set of physically realistic deformation models, which forms a library of canonical functions, including

$$
\begin{aligned}
M_{1}(v(\mathbf{x})) & =t \cdot v(\mathbf{x}) \\
M_{2}(\eta(\mathbf{x})) & =\Delta T \cdot \eta(\mathbf{x}) \\
M_{3}(s(\mathbf{x}), c(\mathbf{x})) & =\sin (2 \pi t) s(\mathbf{x})+(\cos (2 \pi t)-1) c(\mathbf{x}) \\
M_{4}(\kappa(\mathbf{x}), \beta(\mathbf{x})) & =\left(1-\exp \left(-\frac{t}{\beta(\mathbf{x})}\right)\right) \cdot \kappa(\mathbf{x}) \\
M_{5}\left(D_{i}(\mathbf{x})\right) & =D_{i}(\mathbf{x}) \delta_{i j}, \quad i, j \in[1, m] \\
M_{6}\left(\Delta_{i}(\mathbf{x})\right) & =\Delta_{i}(\mathbf{x}) \mathcal{H}\left(t-\tau_{i}(\mathbf{x})\right), \quad i \in[1, m-1]
\end{aligned}
$$

where $M_{q}(\theta)$ represents the $q$ th canonical function of the set of variables $\theta, t$ is the temporal baseline, and $\mathcal{H}\left(t-\tau_{i}(\mathbf{x})\right)(i \in$ $[1, m-1])$ is the Heaviside step function, which is centered at $\tau_{i}(\mathbf{x})$ [36]. $\delta_{i j}$ is the Kronecker delta function of two integer variables $i$ and $j$, which is equal to 1 if the two variables are equal and to 0 otherwise. The library now holds six canonical functions, but this number can be easily extended when other information on the processes is available or when a specific kinematic signature is searched for. The temperaturerelated function can be expressed either by $M_{2}$ or by $M_{3}$. Coefficients $s(\mathbf{x})$ and $c(\mathbf{x})$ in $M_{3}$ represent seasonal periodic deformation with amplitude $A(\mathbf{x})=\sqrt{s^{2}(\mathbf{x})+c^{2}(\mathbf{x})}$ and time offset (w.r.t. the master image time) $t_{0}(\mathbf{x})=-\operatorname{sgn}(c(\mathbf{x}))$. $\arccos (s(\mathbf{x}) / A(\mathbf{x})) / 2 \pi$. The period of the sinusoidal function is here assumed to be one year. Arguments $v(\mathbf{x}), \eta(\mathbf{x}), \kappa(\mathbf{x})$, $\beta(\mathbf{x}), D_{i}(\mathbf{x})$, and $\Delta_{i}(\mathbf{x})$ represent the linear deformation rate, thermal expansion $\eta(\mathbf{x})=\mu_{\Delta T} \cdot L_{\mathrm{LOS}}(\mathbf{x})$ (where $\mu_{\Delta T}$ is the linear expansion coefficient, and $L_{\mathrm{LOS}}$ is the dimension of the object in the line of sight (LOS) direction [37], [38]), the exponential magnitude, the slope factor of the exponential magnitude, an offset at the $i$ th acquisition, and the Heaviside step size. Every offset $D_{i}(\mathbf{x})$ and $\Delta_{i}(\mathbf{x})$ occurs between $t_{i-1}$ and $t_{i}$. In the testing sequence, $M_{5}$ and $M_{6}$ are being evaluated for all positions.

For nonlinear canonical models, such as $M_{4}(\kappa(\mathbf{x}), \beta(\mathbf{x}))$, the model needs to be linearized by a Taylor expansion [39] in order to estimate the parameters.

Many (nested) combinations using these canonical models can be made, and each combination is regarded as a particular
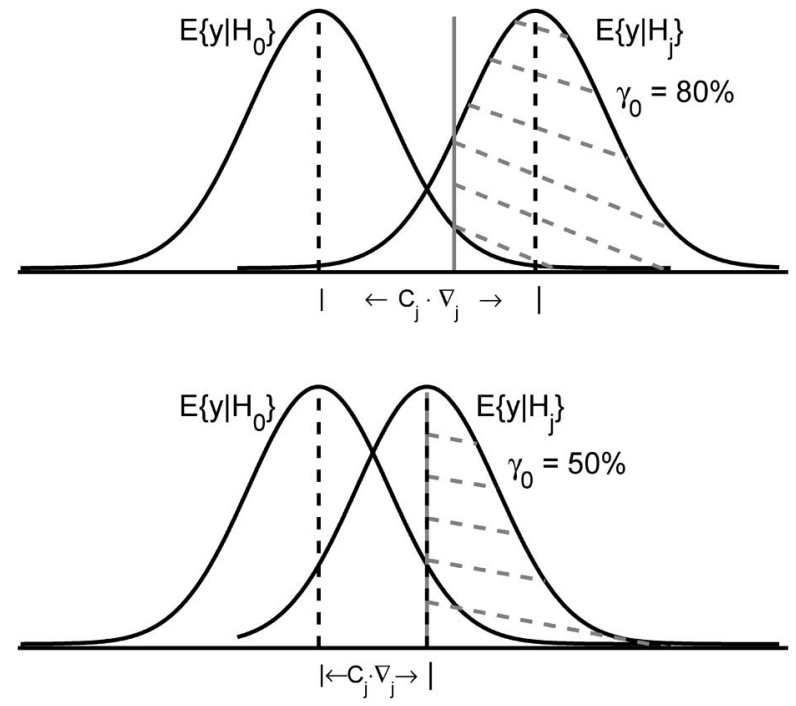

Fig. 1. Discriminatory power $\gamma_{0}$ for an alternative hypothesis $H_{j}$ against null hypothesis $H_{0}$. The estimation difference between $H_{0}$ and $H_{j}$ is equal to $\nabla_{y}=C_{j} \cdot \nabla_{j}$.

alternative hypothesis. Assuming that there may be only one step and only one offset in the time series with $m$ observations, there are already $2 m$ alternative models to be tested. Thus, the amount of $H_{j}$ constructed from the canonical functions can be easily on the order of hundreds.

\section{E. Choice of Test Parameters}

The B-method of testing requires input values for the level of significance, i.e., $\alpha$, and the power of the test, i.e., $\gamma_{0}$ (see Section II-B). To be able to select from different models with the same discriminatory power, particularly when these models have different degrees of freedom, $\alpha$ will need to be tuned. For initializing $\alpha$, we use the $M_{5}$ model with one outlier as a "reference" alternative hypothesis. As a heuristic rule, we choose $\alpha_{0}=1 /(2 m)$, which is related to the number of observations $m$. For InSAR time series, typically, $0.2 \%<\alpha_{0}<2 \%$.

Choosing large discriminatory power, e.g., $\gamma_{0}=80 \%$, implies that an additional parameter $\left|\nabla_{j}\right|$, e.g., the amplitude of a seasonal signal, will need to be large to be detected. A smaller amplitude would be then detected with a smaller likelihood (see Fig. 1). In principle, when comparing different alternative hypotheses, the value for $\gamma_{0}$ is not that important. Here, we choose $\gamma_{0}=50 \%$, which can be interpreted as the size of an additional parameter that would just lead to either the rejection or the acceptance of $H_{0}$.

Given $\alpha_{0}=1 /(2 m), \gamma_{0}=50 \%$, and $q=1$, we then use (8) to compute $\lambda_{0}$, and we invert this again with $q=m-n$ to find the corresponding $\alpha_{G}$ for the OMT. When $m-n$ is a large number, $\alpha_{G}$ will be a large value as well.

\section{F. Procedure for Kinematic Analysis of InSAR Time Series}

With fixed test parameters, the MHT approach can be performed on the kinematic time series. Per point at position $\mathbf{x}=$ $\mathbf{x}(x, y)$, the null hypothesis (steady state) and all alternative 
TABLE I

HyPothesis TABLE LisTing THE COMBINATION OF CANONICAL FUNCTIONS $M_{i}$ FOR EACH HYPOTHESIS $H_{j}$ WITH $q$ DEgREES OF FREEDOM. WHEN AN EXPONENTIAL FUNCTION IS TESTED, IT REPLACES THE LINEAR MODEL $M_{1}$

\begin{tabular}{ccccccccc}
\hline & $H_{0}$ & $H_{1}$ & $H_{2}$ & $H_{3}$ & $H_{4}$ & $H_{5}$ & $H_{6}$ & $H_{7}$ \\
\hline$M_{1}(v(\mathbf{x}))$ & 1 & 1 & 0 & 1 & 0 & 0 & 1 & 0 \\
$M_{2}(\eta(\mathbf{x}))$ & 0 & 1 & 0 & 0 & 1 & 0 & 1 & 1 \\
$M_{4}(\kappa(\mathbf{x}), \beta(\mathbf{x}))$ & 0 & 0 & 1 & 0 & 1 & 1 & 0 & 1 \\
$M_{6}\left(\Delta_{i}(\mathbf{x})\right)$ & 0 & 0 & 0 & 1 & 0 & 1 & 1 & 1 \\
$q$ & & 1 & 2 & 1 & 3 & 3 & 2 & 4 \\
\hline
\end{tabular}

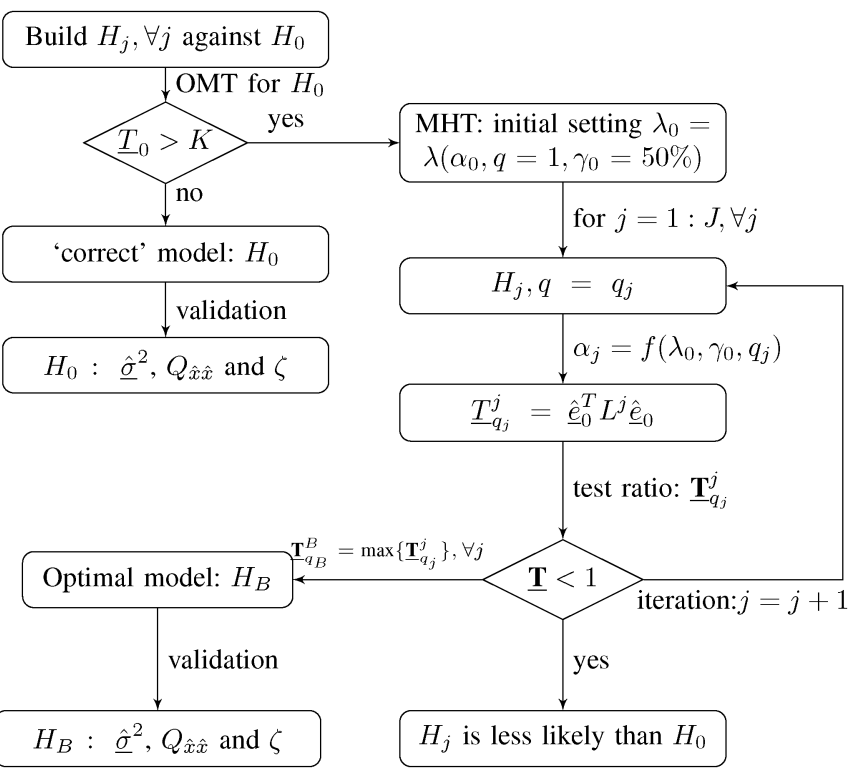

Fig. 2. Testing flowchart for kinematic time-series analysis per point.

hypotheses consisting of various model combinations can be expressed in a binary table (see Table I). Seven alternative hypotheses are listed, whereas the total amount of alternatives, i.e., $J$, is much more than seven since offset $\Delta_{i}(\mathbf{x})$ may happen at any epoch and is evaluated for all epochs. Different combinations for the canonical functions form different alternative hypotheses, e.g., the functional model of $H_{1}$ is the sum of $M_{1}(v(\mathbf{x}))$ and $M_{2}(\eta(\mathbf{x}))$ with one degree of freedom.

Fig. 2 depicts the testing flowchart. We first decide whether to reject or sustain the null hypothesis by using the OMT. Given the level of significance $\alpha_{0}$, the critical value $K$ for the central $\chi^{2}$ distribution is calculated. If $\underline{T}_{0}<K$, there is no need to perform subsequent MHT. In this case, $H_{0}$ is reevaluated in terms of posterior variance $\hat{\sigma}^{2}$, the precision of the parameters $Q_{\hat{x} \hat{x}}$, and eigenvalue $\zeta$ [see (13)].

If $T_{0}>K$, we start the MHT method. The initial noncentrality $\lambda_{0}$ for the given $q=1, \alpha_{0}=1 /(2 m)$, and $\gamma_{0}=50 \%$ is computed and fixed. A loop runs over all $H_{j}$, with $j \in[1, J]$. New $\alpha_{j}$ are used to replace $\alpha_{0}$. Then, test ratios $\underline{\mathbf{T}}_{q j}^{j}(\forall j)$ are calculated based on (6). If $\underline{\mathbf{T}}_{q_{j}}^{j}<1$, the conclusion is that $H_{j}$ is likely not more than $H_{0}$. If $\underline{\mathbf{T}}_{q_{j}}^{j}>1$, the corresponding alternative hypothesis $H_{j}$ is considered a better model candidate and is stored for further use. We then select $\underline{\mathbf{T}}_{q_{B}}^{B}=\max _{j}\left\{\underline{\mathbf{T}}_{q_{j}}^{j}\right\}$ to be the most optimal model for that time series, but we store a number of alternatives for further evaluation. The parameters for optimal model combination $H_{B}$ are then estimated, as well as posterior variance $\underline{\hat{\sigma}}^{2}$, the precision of the parameters $Q_{\hat{x} \hat{x}}$, and eigenvalue $\zeta$.

\section{NUMERICAL IMPLEMENTATION}

To efficiently apply the methodology to a large number of points, we discuss its numerical implementation in this section.

\section{A. Covariance Matrix $Q_{y y}$ and Design Matrix A Under $H_{0}$}

The observation vector in our InSAR time series consists of preprocessed deformation estimates, i.e., per point and per epoch (image acquisition), relative to a reference point and a reference epoch, respectively [40]. The pluriformity of scatterers in space and time makes it impossible to obtain an independent quality metric. Assuming temporal ergodicity and only preselecting values that behave "smooth" in time, we use a fixed variance and assume uncorrelated observations in the time series. Hence, the $m \times m$ covariance matrix $Q_{y y}$ is initialized by the multiplication of a specified variance factor $\sigma^{2}$ and an identity matrix $I_{m}$. This way, the posterior covariance matrix can be estimated by variance component estimation [41]-[44]. When the stochastic structure of the observations is better known beforehand, the covariance matrix can be more precisely specified. The design matrix $A$ under $H_{0}$, as in (1), is a column of temporal baselines with respect to the master acquisition.

\section{B. Preprocessing: RPN Estimation}

In InSAR processing, the kinematic time series of all points are all relative to a common reference point, which implicitly has a postulated zero-displacement time series. However, in reality, the reference point is also a scatterer with instrumental and scattering noise [14] per epoch. Via the differencing operation, the reference point noise (RPN) will manifest itself in every point. In other words, per epoch, the kinematic time series for all points are contaminated by the noise from the reference point. This error can be mitigated. To estimate and eliminate the error of the reference point, we first reduce the deformation time series of every single point by subtracting a linear trend, which is an operation known as temporal deramping. Then, we compute the average value of all points per epoch that is considered to reflect the error of the reference point at that epoch. At the $k$ th epoch, the error of the reference point $e^{k}\left(\mathbf{x}_{\mathrm{rp}}\right)$ is estimated by

$$
\underline{\hat{e}}^{k}\left(\mathbf{x}_{\mathrm{rp}}\right)=\frac{1}{N} \sum_{i=1}^{N} \underline{e}_{i}^{k}, \quad \forall k
$$

where $N$ denotes the number of points, and $e_{i}^{k}$ represents the residual for the $i$ th point after temporal deramping at the $k$ th epoch. This error is subsequently subtracted from the kinematic time series for all points, i.e.,

$$
\underline{y}_{i}^{\prime}=\underline{y}_{i}-\underline{\hat{e}}\left(\mathbf{x}_{\mathrm{rp}}\right), \quad \forall i
$$

which yields an RPN-reduced time series $\underline{y}^{\prime}$, which is to be used in the subsequent hypothesis testing approach. Informally, we refer to this RPN reduction method as the Shenzhen algorithm due to the location where this idea sprouted. 

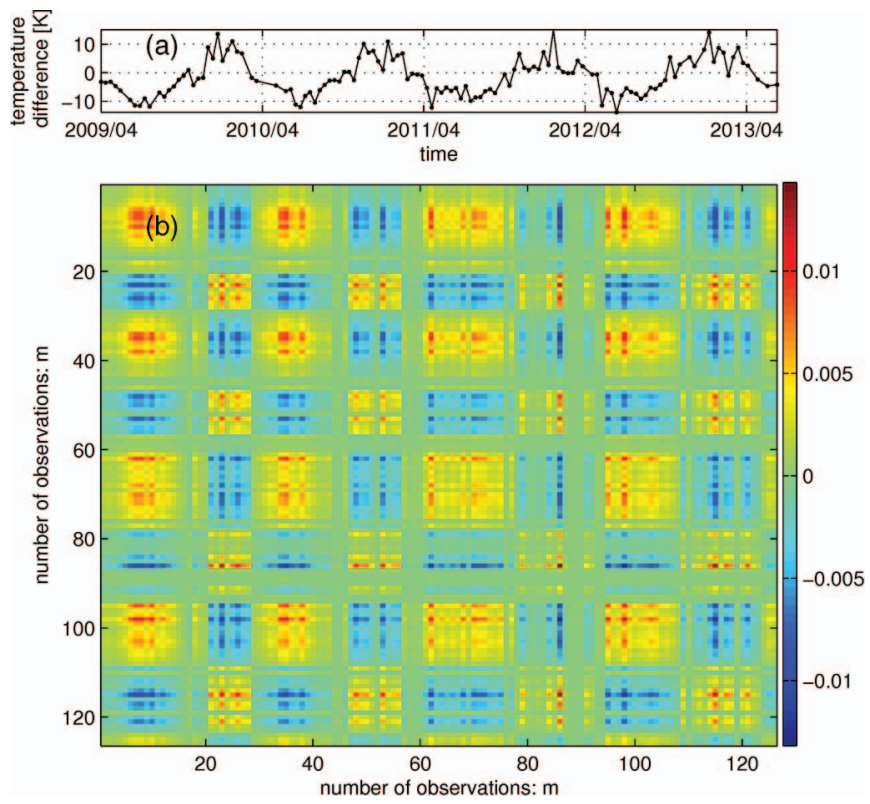

Fig. 3. (a) Temperature differences between the acquired dates of the TerraSAR-X master image and the slaves. (b) $L$ matrix for the thermal expansion with arbitrary units [cf., (5)].

\section{Preprocessing: Detecting Unwrapping Errors}

After time-series InSAR processing, there are still points that exhibit the remaining unwrapping errors either as an individual $2 \pi$ outlier or as a "cycle slip," where two parts of the time series show a $2 \pi$ offset. To detect the remaining unwrapping errors, the likelihood of the remaining $2 \pi$ ambiguities in the kinematic time series is tested using the same MHT procedure [see (14)] by fixing outlier $D_{i}(\mathbf{x})$ and offset $\Delta_{i}(\mathbf{x})$ to be $\pm \lambda_{\mathrm{SAR}} / 2$, where $\lambda_{\text {SAR }}$ represents the SAR wavelength. When hypothesis testing yields $\left|D_{i}(\mathbf{x})\right|$ or $\left|\Delta_{i}(\mathbf{x})\right|$ larger than $\left|\lambda_{\mathrm{SAR}} / 4\right|$, the observation vector for that point will be corrected by $\pm \lambda_{\mathrm{SAR}} / 2$ to clean it from unwrapping errors before subsequent hypothesis testing for other model errors.

\section{Efficient Test Statistic Computation}

A testing procedure with hundreds of alternative hypotheses for millions of InSAR points would be numerically very inefficient. As stated in Section II-A, the computational burden is significantly reduced by expressing the test statistic for alternative hypothesis $j$ as

$$
\underline{T}_{q}^{j}=\underline{\hat{e}}_{1 \times m}^{T} \underset{m \times m}{L^{j}} \underset{m \times 1}{\underline{\hat{e}}_{0}}=\operatorname{tr}\left(\begin{array}{ccc}
L_{m \times m}^{j} & \underline{\hat{e}}_{0 \times 1} & \hat{\underline{e}}_{0}^{T} \\
\frac{1 \times m}{1 \times m}
\end{array}\right)
$$

where the $L^{j}$ matrix needs to be computed once for every hypothesis class, but it is equal for each point; thus, it can be retrieved in internal memory. The $m \times m$ matrix $\underline{\hat{e}}_{0} \underline{\hat{e}}_{0}^{T}$ is only computed once per point, i.e., in only evaluating the null hypothesis.

For instance, when only the thermal expansion is considered an additional component in the functional model [cf., $M_{2}$ in (14)], the corresponding $L$ matrix is temperature related. Fig. 3(a) shows the temperature differences between the ac- quired dates of the TerraSAR-X master image (April 8, 2009) and the slaves using 127 satellite images. The corresponding $126 \times 126$ matrix $L$ is shown in Fig. 3(b). The seasonal variation of the temperature changes is clearly shown in Fig. 3(b) as the color changes from red to blue. This $L$ matrix will be further used in the real case study over Rotterdam (see Section IV-B).

\section{Application on Simulated Data and Real Data}

\section{A. Application on Simulated Data}

To demonstrate the feasibility of kinematic model selection and parameter estimation using a probabilistic approach, we evaluate a synthetic example. We simulate 70 acquisitions by the Envisat satellite with a $5.62-\mathrm{cm}$ wavelength between December 2003 and October 2010 for a single point relative to an arbitrary reference point. We use real temperature records for the actual Envisat acquisition times. The time series of this point is modeled as a superimposition of linear motion $(v=$ $-10 \mathrm{~mm} /$ year), thermal expansion $\eta=+1.3 \cdot 10^{-3} \mathrm{~m} \cdot \mathrm{K}^{-1}$, and an instantaneous Heaviside offset at or before the 26th acquisition with a level of $\Delta_{26}=-18 \mathrm{~mm}$. Gaussian-distributed random noise with a standard deviation of $5 \mathrm{~mm}$ is added. Hence, this fits with the sixth alternative hypothesis model $H_{6}$, as in Table I [see Fig. 4(a)]. Following the processing procedure in Section II-F, the OMT is first applied for null hypothesis $H_{0}$ under the level of significance $\alpha_{G}=23 \%$, which follows via the B-method of testing from $\alpha_{0}=1 /(2 m)$ for the 1-D test (see Section II-E). The test statistic corresponding to each model is listed above the graphs in Fig. 4(a)-(h). For $H_{0}$, the value is much larger than the critical value $K$ [see Fig. 4(a)], causing its rejection. Then, the procedure starts to find the optimal model among the alternatives. After evaluating every test statistic ratio, which corresponds with Fig. 4(a)-(h), the most optimal model with the greatest test statistic ratio is the alternative hypothesis $H_{6}$. For model $H_{6}$, we then estimate the corresponding parameters $\hat{v}, \hat{\eta}$, and $\hat{\Delta}$ to be $-10.5 \mathrm{~mm} /$ year, $+1.4 \cdot 10^{-3} \mathrm{~m} \cdot \mathrm{K}^{-1}$, and $-17.1 \mathrm{~mm}$ at the 26 th acquisition, respectively, which are close enough to the simulated values. After determining that selection model combination $H_{6}$ is the most optimal model, the posterior variance for $H_{6}$ is estimated to be $4.5^{2} \mathrm{~mm}^{2}$.

\section{B. Application on Real Data}

To test the approach using real data, an experiment is performed over Rotterdam, the Netherlands, from April 2009 to June 2013. We use 127 TerraSAR-X stripmap images and apply the Delft implementation of PS Interferometry (DePSI) [10] to produce the kinematic time series of PSs using a default steadystate model to facilitate temporal unwrapping.

We regard these PS results as the input data for kinematic time-series modeling. We remove the RPN from the data using the Shenzhen algorithm (see Section III-B). We use the daily average temperature data for every acquired date [see Fig. 3(a)]. The variance of unit weight $\sigma^{2}$, as in (1), is conservatively predefined as $3^{2} \mathrm{~mm}^{2}$, and cofactor matrix $R_{y y}$ is designed as an identity matrix. With a $31-\mathrm{mm}$ wavelength, $\sigma=3 \mathrm{~mm} \approx 1.2 \mathrm{rad}$. 

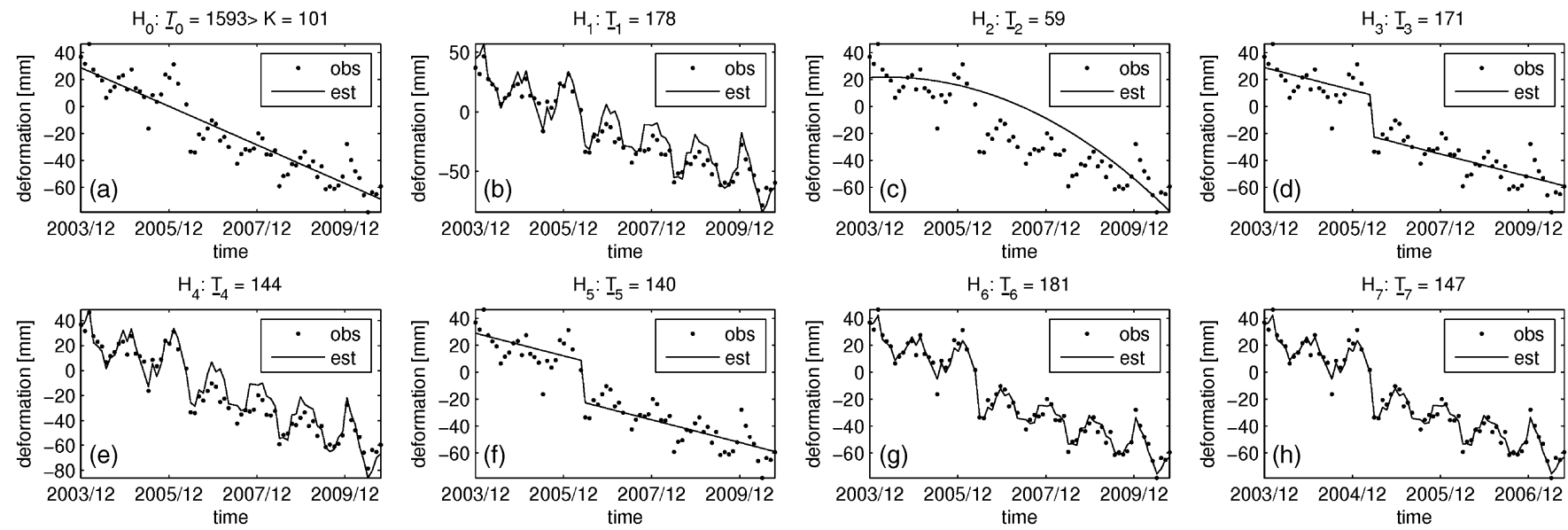

Fig. 4. Synthetic example for MHT. The black dots show the simulated kinematic data with the noise modeled as the sixth alternative hypothesis model $H_{6}$. (a) Null hypothesis model: steady-state (linear) motion, which is the default model for InSAR processing. (b)-(h) Estimated parameters for alternative hypotheses $H_{1}-H_{7}$, respectively. Based on test statistic ratios $\underline{\mathbf{T}}_{q_{k}}^{k}$, which are printed above each graph, the most probable model is determined, i.e., alternative hypothesis $H_{6}$ that has the greatest test statistic ratio.
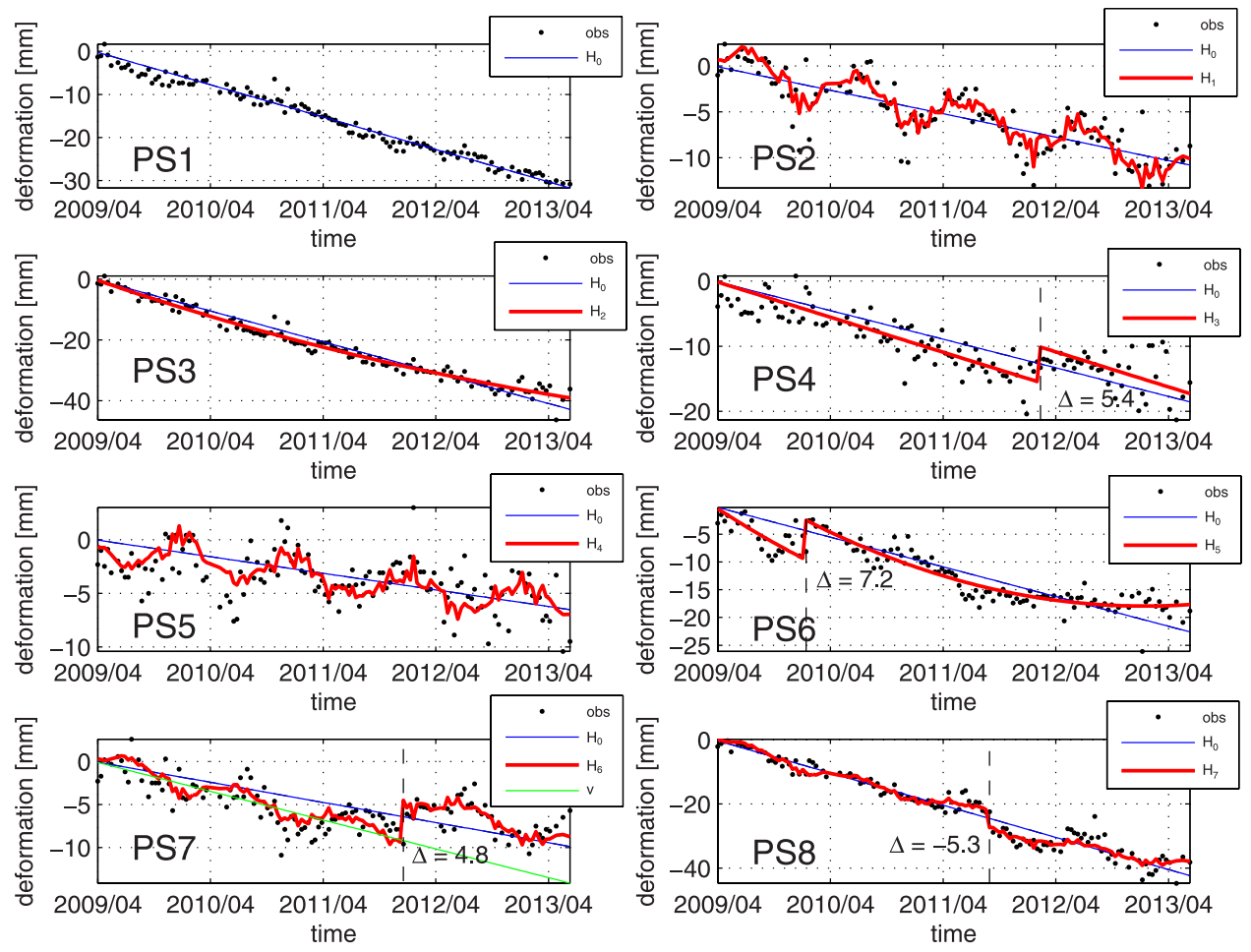

Fig. 5. Real data examples for MHT. Each subfigure shows the optimal deformation model found for a randomly selected PS point (see Fig. 7(a) and (b) for the location).

The results from the kinematic time-series modeling show that $84 \%$ of the points sustain the steady-state null hypothesis $H_{0}$, whereas the others reject $H_{0}$ and follow the alternative models. Fig. 5 illustrates the MHT results for eight typical PS points, and the parameter estimates for their optimal models are listed in Table II. The locations of the points are shown in Fig. 7. These results show that: 1) each function from the library of canonical kinematic models seems physically realistic and can be used to produce an optimal kinematic model for these real data; 2) the optimal models and the quality of estimates are different per point; and 3) the optimal models for the eight PS points fit the kinematic time series (black dots) better than the linear deformation model (blue lines) in a probabilistic sense. All but one of the posterior variances $\underline{\hat{\sigma}}^{2}$ of the eight points shown in Table II are smaller than the conservative a priori variances, which improves the reported quality of the estimates and the time series. In addition, the kinematic timeseries results allow us to detect anomalous offsets $\Delta$ for PS4, PS6, PS7, and PS8.

Fig. 6 demonstrates that the MHT method is able to fix unnoticed unwrapping errors. It shows the MHT results for PS points 6, 9, and 10 (cf., Fig. 7). $H_{5}, H_{3}$, and $H_{6}$ (black lines) are found to be the optimal models for them, respectively. For PS6, a Kronecker delta outlier is found at the 114th epoch, which is greater than the half-wavelength, i.e., $15.5 \mathrm{~mm}$ [see the arrow indicator in Fig. 6(a)]. Thus, the algorithm identifies it as the 
TABLE II

Kinematic Time-Series Modeling Results for Eight PS Points (PS1-PS8) (SEe Fig. 5)

\begin{tabular}{|c|c|c|c|c|c|c|c|c|c|}
\hline Point ID & Model Nr. & $\begin{array}{c}\hat{v} \\
{\left[\mathrm{~mm} \cdot \mathrm{y}^{-1}\right]}\end{array}$ & {$\left[10^{-3} \stackrel{\hat{\eta}}{\left.\mathrm{m} \cdot \mathrm{K}^{-1}\right]}\right.$} & $\hat{\kappa}$ & $\hat{\beta}$ & $\begin{array}{r}\hat{\Delta} \\
{[\mathrm{mm}]}\end{array}$ & @ acq.date & var.factor & $\frac{\hat{\sigma}}{[\mathrm{mm}]}$ \\
\hline PS1 & $H_{0}$ & -7.2 & & & & & & 0.2 & 1.3 \\
\hline PS2 & $H_{1}$ & -2.6 & -0.24 & & & & & 0.6 & 2.3 \\
\hline PS3 & $H_{2}$ & & & -78.3 & +6.0 & & & 0.4 & 1.9 \\
\hline PS4 & $H_{3}$ & -5.4 & & & & +5.4 & (28-Feb-2012) & 1.1 & 3.1 \\
\hline PS5 & $H_{4}$ & & +0.18 & -21.2 & +12.6 & & & 0.4 & 1.9 \\
\hline PS6 & $H_{5}$ & & & -30.0 & +2.2 & +7.2 & (30-Jan-2010) & 0.6 & 2.3 \\
\hline PS7 & $H_{6}$ & -3.4 & -0.21 & & & +4.8 & (04-Jan-2012) & 0.5 & 2.1 \\
\hline PS8 & $H_{7}$ & & -0.19 & -10.6 & +3.1 & -5.3 & (16-Sep-2011) & 0.6 & 2.3 \\
\hline
\end{tabular}

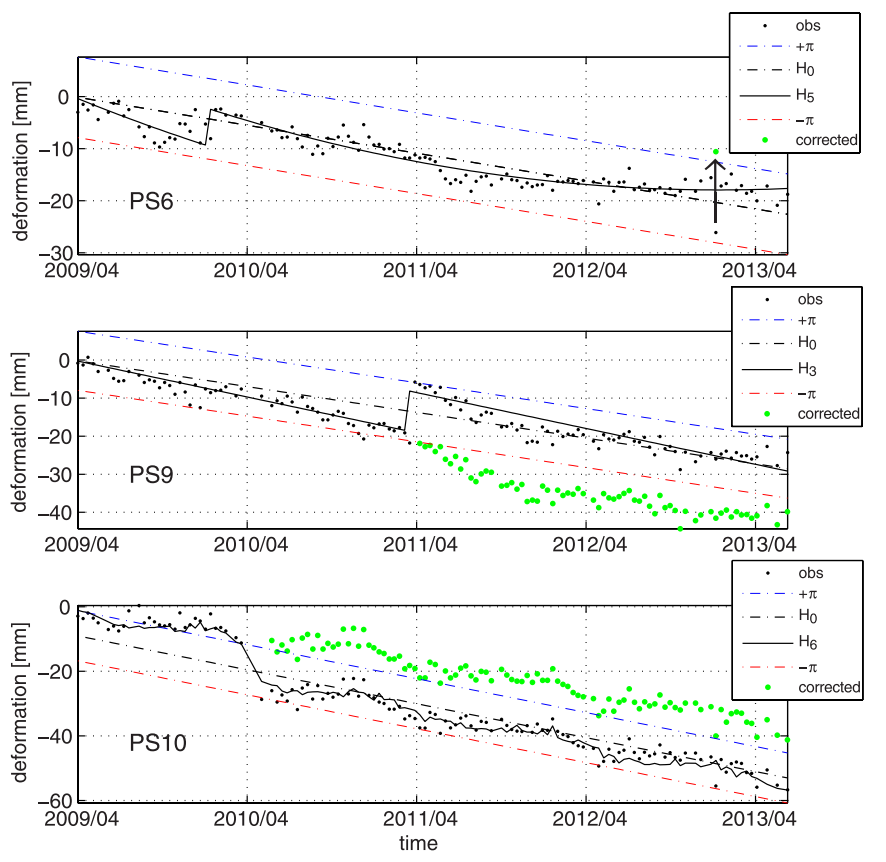

Fig. 6. Examples for residual unwrapping error detection and correction by the MHT approach. The black up arrow in the top plot indicates the observation on January 23, 2013, which is corrected for the unwrapping error from the black dot to the green dot. The lower plots indicate the detected offsets (cycle slips) in the observations.

single unwrapping error on January 23, 2013 and corrects it (indicated by the green dot). For PS9 and PS10, the values of the estimated Heaviside offsets are larger than $15.5 \mathrm{~mm}$ as well. Therefore, they are identified as residual unwrapping errors, and they are corrected by adding the offsets back (shown in green).

To demonstrate the applicability of our approach on large volumes of data and analyze the spatial behavior of data, we apply kinematic time-series modeling on a $4.5 \mathrm{~km} \times 13 \mathrm{~km}$ area near Rotterdam, the Netherlands, with 748806 PS points. Fig. 7(a) and (b) shows the ground features of a subset $(0.5 \mathrm{~km} \times 2 \mathrm{~km})$ of this crop area, which dominantly is a railway (Betuweroute, which is denoted by "B") and a motorway (A15) with some adjacent buildings. This subset shows 5857 detected PSs, of which 10 points are further elaborated in time (see Figs. 5 and 6), as indicated by the red crosses.

Fig. 7(c) shows the LOS velocity map of all PS points selected by the default PSI processing. It indicates that the PS points reflected from the buildings [in yellow in Fig. 7(b)] are stable, whereas the PS points reflected from the railway lines and the motorway are deforming but with different rates. Along the railway lines, the PS velocities are heterogeneous, e.g., the discrete discontinuities of the velocities are detected in the transition zones between free embankments and fixed substructures such as the bridge [cf., C in Fig. 7(b)]. Fig. 7(c) shows the LOS velocity map of $84 \%$ of the PS points that sustain null hypothesis $H_{0}$. That is, the motion of most PSs on buildings, bridges, and the railway is best described by the default steadystate model. Yet, Fig. 7(e) shows the thermal expansion parameter $\eta$ of those PSs whose optimal model includes $M_{2}(\eta(\mathbf{x}))$ [cf., (14)]. It suggests that the motions of the ground targets made of concrete or steel, such as buildings, railway lines, and lamp poles, are subject to the temperature variations in the form of expansion when the temperature rises and in the form of contraction when the temperature falls. Consequently, the thermal expansion parameter for these targets is negative $(\eta<0)$, as shown in orange/red in this figure. For instance, building A, bridge $\mathrm{C}$, and the railway parts and segments made of concrete/ steel (e.g., PS2, PS7, and PS8) all have a negative thermal expansion parameter. The data also show the motion of targets with a positive thermal expansion parameter (in blue). These behave conversely, such as the motion of some segments of the railway tracks and a graveyard area denoted by D and PS5. These are most likely influenced by compaction due to seasonal changes in the groundwater table. Thus, according to the value (and sign) of the thermal expansion parameter, we can classify the PS points in terms of different materials and characteristics. Areas where a sign change occurs [cf., the rectangular black box in Fig. 7(e)] may signal the susceptibility of locations and materials to fatigue. Fig. 7(f) shows the LOS velocity map derived from the postdetermined best models for the PS points shown in Fig. 7(e). It shows that buildings and bridges (e.g., building $\mathrm{A}$ and bridge $\mathrm{C}$ ) experience minimal motion apart from those that are temperature related, whereas the railway tracks show heterogeneous motion at different rail segments, which is probably due to different local situations such as consolidated/unconsolidated soils. Fig. 7(g) shows the estimated velocity differences between $H_{0}$ and the postdetermined best models for the PS points shown in Fig. 7(e). It implies that if we harshly stick to null hypothesis $H_{0}$ for every single point, we may overestimate or underestimate the linear deformation, leading to an erroneous interpretation of the time series. For example, the deformation rate of PS7 under $H_{6}$ (including thermal variability) is $v_{H_{6}}=-3.4 \mathrm{~mm} / \mathrm{year}$ (the green line in Fig. 5), which is larger than the rate under $H_{0}$, i.e., $v_{H_{0}}=$ $-2.4 \mathrm{~mm} /$ year (see also Table II). The fact that the likelihood of $H_{6}$ is greater than that of $H_{0}$ now improves the reliability of the estimates. 
(a)
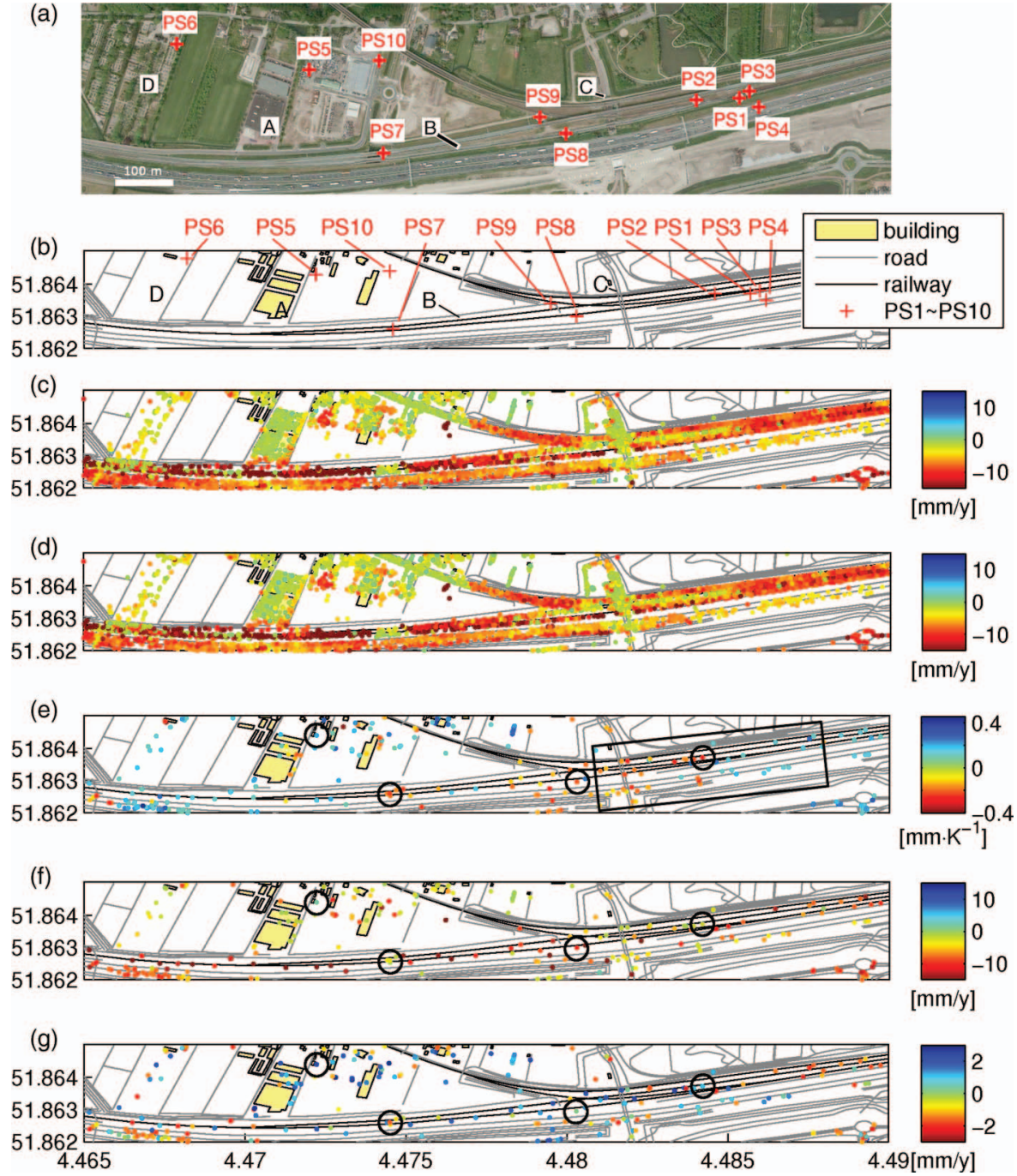

Fig. 7. Kinematic time-series analysis for the $0.5 \mathrm{~km} \times 2 \mathrm{~km}$ test area. (a) Aerial image (Bing maps). (b) Cartographic representation (TOP10vector, 2011). (c) Default LOS velocity map resulting from standard PSI processing. (d) Points that sustain the null hypothesis of steady-state deformation only. (e) Thermal expansion parameter $\eta$ of those points whose optimal model combination includes $M_{2}(\eta(\mathbf{x})$ ). (f) LOS velocity map corresponding to the points shown in (e). (g) Estimated velocity differences between $H_{0}$ [cf., (c)] and the postdetermined best models for the points shown in (e).

In terms of computational efficiency, since our method does not require the explicit estimation and adjustment of all the parameters for each evaluated model, the computational load is reduced with a factor of 5 . The computation time for the test is $31 \mathrm{~ms}$ per point, testing about 508 alternative hypotheses on an average desktop computer.

\section{DisCUSSION AND CONCLUSIONS}

A probabilistic approach using MHT and the B-method of testing is proposed for kinematic time-series modeling and parameter estimation. It is generally applicable to select the optimal models for single points in InSAR kinematic time series and to detect and correct phase unwrapping errors in a probabilistic sense. This way, apart from precision, the geodetic concept of reliability is now also added to InSAR estimates, which is defined as the ability to detect observational errors or model imperfections from the data. These model imperfections can relate to both the functional and stochastic models, with the latter giving an improved quality description of the results. Although there may be several competing models with a high likelihood of being true, the methodology will allow users to critically evaluate results, avoid overinterpretation, and thereby consolidate InSAR as a geodetic technique.

\section{REFERENCES}

[1] A. Ferretti, C. Prati, and F. Rocca, "Nonlinear subsidence rate estimation using permanent scatterers in differential SAR interferometry," IEEE Trans. Geosci. Remote Sens., vol. 38, no. 5, pp. 2202-2212, Sep. 2000.

[2] A. Ferretti, C. Prati, and F. Rocca, "Permanent scatterers in SAR interferometry," IEEE Trans. Geosci. Remote Sens., vol. 39, no. 1, pp. 8-20, Jan. 2001.

[3] P. Berardino, G. Fornaro, R. Lanari, and E. Sansosti, "A new algorithm for surface deformation monitoring based on small baseline differential SAR interferograms," IEEE Trans. Geosci. Remote Sens., vol. 40, no. 11, pp. 2375-2383, Nov. 2002.

[4] B. Kampes, "Displacement parameter estimation using permanent scatterer interferometry," DLR-Inst. Aerosp. Med., Braunschweig, Germany, Forschungsbericht. DLR, Bibliotheks- und Informationswesen, 2005.

[5] P. Tizzani et al., "Surface deformation of Long Valley caldera and Mono Basin, California, investigated with the SBAS-InSAR approach," Remote Sens. Environ., vol. 108, no. 3, pp. 277-289, Jun. 2007.

[6] A. Hooper, P. Segall, and H. Zebker, "Persistent scatterer interferometric synthetic aperture radar for crustal deformation analysis, with application to Volcán Alcedo, Galápagos," J. Geophys. Res., vol. 112, no. B7, 2007, Art. ID. B07407. 
[7] A. Hooper, "A multi-temporal InSAR method incorporating both persistent scatterer and small baseline approaches," Geophys. Res. Lett., vol. 35, no. 16, pp. 1-5, Aug. 2008.

[8] R. Lanari et al., "Surface displacements associated with the L'Aquila 2009 Mw 6.3 earthquake (central Italy): New evidence from SBAS-DInSAR time series analysis," Geophys. Res. Lett., vol. 37, no. 20, Oct. 2010, Art. ID. L20309.

[9] S. Usai and R. Klees, "SAR interferometry on very long time scale: A study of the interferometric characteristics of man-made features," IEEE Trans. Geosci. Remote Sens., vol. 37, no. 4, pp. 2118-2123, Jul. 1999.

[10] F. van Leijen, "Persistent scatterer interferometry based on geodetic estimation theory," Netherlands Geodetic Commission, Amersfoort, The Netherlands, no. 86, 2014

[11] W. Xu, and I. Cumming, "A region-growing algorithm for InSAR phase unwrapping," IEEE Trans. Geosci. Remote Sens., vol. 37, no. 1, pp. 124-134, Jan. 1999

[12] R. F. Hanssen, Radar Interferometry: Data Interpretation and Error Analysis. Dordrecht, The Netherlands: Kluwer, 2001.

[13] H. A. Zebker and J. Villasenor, "Decorrelation in interferometric radar echoes," IEEE Trans. Geosci. Remote Sens., vol. 30, no. 5, pp. 950-959, Sep. 1992.

[14] H. A. Zebker, P. A. Rosen, R. M. Goldstein, A. Gabriel, and C. L. Werner "On the derivation of coseismic displacement fields using differential radar interferometry: The Landers earthquake," J. Geophys. Res., vol. 99, no. B10, pp. 19617-19634, Oct. 10, 1994.

[15] C. Tiberius, "Recursive data processing for kinematic GPS surveying," Nederlandse Commissie Voor Geodesie, Amersfoort, The Netherlands, 1998, no. 45 .

[16] P. J. G. Teunissen, D. G. Simons, and C. C. J. M. Tiberius, "Probability and observation theory," Delft Inst. Earth Observ. Space Syst. (DEOS), Delft Univ. Technol., Delft, The Netherlands, 2005.

[17] A. Ferretti et al., "A new algorithm for processing interferometric datastacks: SqueeSAR," IEEE Trans. Geosci. Remote Sens., vol. 49, no. 9, pp. 3460-3470, Sep. 2011

[18] A. Hooper, H. Zebker, P. Segall, and B. Kampes, "A new method for measuring deformation on volcanoes and other non-urban areas using InSAR persistent scatterers," Geophys. Res. Lett., vol. 31, Dec. 2004 Art. ID. L23611.

[19] M. Caro Cuenca, "Improving radar interferometry for monitoring faultrelated surface deformation: Applications for the Roer Valley Graben and coal mine induced displacements in the Southern Netherlands," Netherlands Geodetic Commission, Delft, The Netherlands, no. 81, 2012.

[20] L. Chang and R. F. Hanssen, "Detection of cavity migration and sinkhole risk using radar interferometric time series," Remote Sens. Environ., vol. 147, pp. 56-64, May 2014.

[21] F. Chen, H. Lin, Z. Li, Q. Chen, and J. Zhou, "Interaction between permafrost and infrastructure along the Qinghai-Tibet Railway detected via jointly analysis of C- and L-band small baseline SAR interferometry," Remote Sens. Environ., vol. 123, pp. 532-540, Aug. 2012.

[22] C. Xie, Z. Li, J. Xu, and X. Li, "Analysis of deformation over permafrost regions of Qinghai-Tibet plateau based on permanent scatterers," Int. J. Remote Sens., vol. 31, no. 8, pp. 1995-2008, 2010.

[23] J. J. Sousa, A. J. Hooper, R. F. Hanssen, L. C. Bastos, and A. M. Ruiz, "Persistent scatterer InSAR: A comparison of methodologies based on a model of temporal deformation vs. spatial correlation selection criteria," Remote Sens. Environ., vol. 115, no. 10, pp. 2652-2663, Oct. 2011

[24] K. Koch, Parameter Estimation and Hypothesis Testing in Linear Models. New York, NY, USA: Springer-Verlag, 1988.

[25] P. J. G. Teunissen, Testing Theory: An Introduction, 1st ed. Delft, The Netherlands: Delft Univ. Press, 2000.

[26] W. Baarda, A Testing Procedure for Use in Geodetic Networks, 2nd ed., ser. Publications on Geodesy. Delft, The Netherlands: Netherlands Geodetic Commission, 1968.

[27] H. Akaike, "A new look at the statistical model identification," IEEE Trans. Autom. Control, vol. 19, no. 6, pp. 716-723, Dec. 1974.

[28] H. Akaike, "On the likelihood of a time series model," Statistician, vol. 27 , no. 3/4, pp. 217-235, Sep.-Dec. 1978.

[29] P. Stoica and Y. Selen, "Model-order selection: A review of information criterion rules," IEEE Signal Process. Mag., vol. 21, no. 4, pp. 36-47, Jul. 2004.

[30] P. J. G. Teunissen, "Quality control in integrated navigation systems," IEEE Aerosp. Electron. Syst. Mag., vol. 5, no. 7, pp. 35-41, Jul. 1990.

[31] H. M. de Heus, P. Joosten, M. H. F. Martens, and H. M. E. Verhoef, "Geodetische deformatie analyse: 1Ddeformatieanalyse uit waterpasnetwerken," Delft Univ. Technol., LGR Series, Delft, The Netherlands, Tech. Rep. 5, 1994.
[32] P. J. Davis, "Gamma function and related functions," in Handbook of Mathematical Functions, 9th ed., ser. Dover books on advanced mathematics. New York, NY, USA: Dover, 1970, ch. 6, pp. 253-294.

[33] M. Abramowitz and I. A. Stegun, Handbook of Mathematical Functions, 9th ed., ser. Dover books on advanced mathematics. New York, NY, USA: Dover, 1970.

[34] P. J. G. Teunissen, "Integer aperture GNSS ambiguity resolution," Artif. Satellites, vol. 38, no. 3, pp. 79-88, 2003.

[35] W. Baarda, S-Transformations and Criterion Matrices, 2nd ed., ser. Publications on Geodesy, New Series. Delft, The Netherlands: Netherlands Geodetic Commission, 1981.

[36] E. W. Weisstein, Heaviside Step Function. Champaign, IL, USA: MathWorld-A Wolfram Web Resource, 2008.

[37] O. Monserrat, M. Crosetto, M. Cuevas, and B. Crippa, "The thermal expansion component of persistent scatterer interferometry observations," IEEE Geosci. Remote Sens. Lett., vol. 8, no. 5, pp. 864-868, Sep. 2011.

[38] M. Cuevas, O. Monserrat, M. Crosetto, and B. Crippa, "A new product from persistent scatterer interferometry: The thermal dilation maps," in Proc. IEEE JURSE, 2011, pp. 285-288.

[39] T. G. Goodwillie, "Calculus III: Taylor series," Geometry Topology, vol. 7, no. 2, pp. 645-711, 2003.

[40] R. Hanssen, "Stochastic modeling of time series radar interferometry," in Proc. IEEE Int. Geosci. Remote Sens. Symp., Anchorage, AK, USA, 2004 pp. 2607-2610.

[41] P. J. G. Teunissen, Towards a Least-Squares Framework for Adjusting and Testing of Both Functional and Stochastic Models, ser. Mathematical Geodesy and Positioning Series. Delft, The Netherlands: Delft Univ. Press, 1988

[42] F. Kenselaar, "Model formulation and adjustment of geodetic positioning problems," Delft Univ. Technol., Delft, The Netherlands, Tech. Rep., 1997.

[43] A. Amiri-Simkooei, C. Tiberius, and P. Teunissen, "Assessment of noise in GPS coordinate time series: Methodology and results," J. Geophys. Res.: Solid Earth (1978-2012), vol. 112, no. B7, Jul. 2007, Art. ID. B07413.

[44] P. J. G. Teunissen and A. R. Amiri-Simkooei, "Least-squares variance component estimation," J. Geodesy, vol. 82, no. 2, pp. 65-82, Feb. 2008.

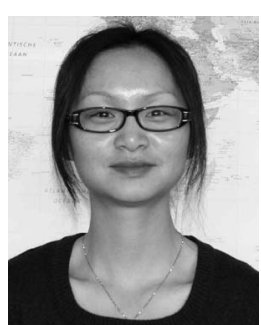

Ling Chang received the M.S.E. degree in geodesy and survey engineering from Tongji University, Shanghai, China, in 2010 and the Ph.D. degree from the Delft University of Technology, Delft, The Netherlands, in 2015

She is currently with the Department of Geoscience and Remote Sensing, Delft University of Technology. Her studies regard new algorithms in semirecursive estimation and the kinematic timeseries modeling of interferometric synthetic aperture radar measurement points. Her current research interests include statistical hypothesis testing, the structural health monitoring of infrastructure, and anomalous change detection in near real time using satellite radar interferometry.

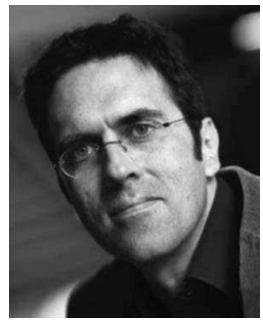

Ramon F. Hanssen (M'04-SM'15) received the M.Sc. degree in geodetic engineering and the Ph.D. degree (cum laude) from the Delft University of Technology, Delft, The Netherlands, in 1993 and 2001 , respectively.

$\mathrm{He}$ was with the International Institute for Aerospace Survey and Earth Science (ITC); Stuttgart University; the German Aerospace Center (DLR); Stanford University (Fulbright Fellow); and Scripps Institution of Oceanography where he worked on microwave remote sensing, radar interferometry, signal processing, and geophysical application development. Since 2008, he is Antoni van Leeuwenhoek Professor in Earth Observation at Delft University of Technology, where he has been leading the Department of Geoscience and Remote Sensing since 2011. He is the author of a textbook on radar interferometry. 